This is the accepted manuscript of the article, which has been published in Spectrochimica Acta Part A:

Molecular and Biomolecular Spectroscopy . 2019, 218, 299-307. https://doi.org/10.1016/j.saa.2019.04.010

\title{
Identifying yeasts using surface enhanced Raman spectroscopy
}

\section{Tibebe Lemma ${ }^{1, *}$, Jin Wang ${ }^{2}$, Kai Arstila ${ }^{3}$, Vesa P. Hytönen ${ }^{4,}$ and J. Jussi Toppari ${ }^{3}$}

1 Faculdade de Clências e Tecnologia (FCT)-Universidade Estadual Paulista (UNESP)-Presidente Prudente, SP,19060-900, Brazil

${ }^{2}$ Institute of Intelligent Machines, Chinese Academy of Sciences, Hefei, Anhui 230031, P. R. China

3 NanoScience Center, Department of Physics, P.O. Box 35 (YN), FI-40014, University of Jyväskylä, Finland

4 Faculty of Medicine and Health Technology and BioMediTech, Tampere University, Arvo Ylpön katu 34, FI-33520 Tampere, Finland and Fimlab Laboratories, Biokatu 4, FI-33520 Tampere, Finland

* Correspondence: tlemma@gmail.com (T.L.)

\begin{abstract}
The molecular fingerprints of yeasts Saccharomyces cerevisiae, Dekkera bruxellensis, and Wickerhamomyces anomalus (former name Pichia anomala) have been examined using surfaceenhanced Raman spectroscopy (SERS) and helium ion microscopy (HIM). The SERS spectra obtained from cell cultures (lysate and non-treated cells) distinguish between these very closely related fungal species. Highly SERS active silver nano-particles suitable for detecting complex biomolecules were fabricated using a simple synthesis route. The yeast samples mixed with aggregated Ag nanoparticles yielded highly enhanced and reproducible Raman signal owing to the high density of the hot spots at the junctions of two or more Ag nanoparticles and enabled to differentiate the three species based on their unique features (spectral fingerprint). We also collected SERS spectra of the three yeast species in beer medium to demonstrate the potential of the method for industrial application. These findings demonstrate the great potential of SERS for detection and identification of fungi species based on the biochemical compositions, even in a chemically complex sample.
\end{abstract}

Keywords: Silver nanoparticle; aggregate; SERS; yeast; helium ion microscopy

\section{Introduction}

Yeast is the most important part of the brewing fermentation process. Yeasts, such as Saccharomyces cerevisiae (baker's yeast or brewer's yeast) and Dekkera bruxellensis (also known as Brettanomyces bruxellensis) are commonly used in food production, baking, wine, and beer making. Preparations of baker's yeasts are also used as dietary, nutrient supplements because of their high contents of B vitamins, proteins, peptides, amino acids, and trace minerals. In addition to fermenting and flavoring foods, baker's yeast also plays an important role as a model organism in the field of biochemistry, genetics and molecular biology, and it is important commercially and industrially [1]. Yeasts can also be used as an oral, biotherapeutic agent to treat patients with severe cases of diarrhea and other gastrointestinal 
disorders [2]. However, yeasts are also responsible for the majority of food and beverage spoilage, which brings on their enormous commercial and economic significance. While there is significant diversity in the yeast species associated with food and beverage spoilage, Saccharomyces cerevisiae (wild yeast), Dekkera bruxellensis and Wickerhamomyces anomalus (former name Pichia anomala) are responsible for the vast majority of spoilage in the winery in cases not intentionally used in the fermentation process [3].

Most of the organisms in the fungi kingdom are multicellular, however, yeast, a typical fungus, is a single-celled eukaryote organism, and thus it contains membrane-bound organelles, such as a nucleus, endomembrane system, and mitochondria. A yeast cell is surrounded by a rigid wall which is $\sim 250 \mathrm{~nm}$ thick and constituting about $15-30 \%$ of the total dry mass of the cell $[4,5]$. The cell wall of yeast is an important component which is responsible for mechanical strength (barrier) of the cell. The wall is composed of mannoprotein [1, 6], glucan, beta-glucan, and chitin (a long-chain polymer of an $\mathrm{N}$ acetylglucosamine), which contributes to the insolubility of the fibers. In addition, some proteins are disulfide-bonded to other cell wall proteins [6] which make yeast cell highly diverse in their protein and other components. A single unbudded yeast cell is about 5-10 $\mu \mathrm{m}$ in diameter and is usually oval, cylindrical or spherical in shape.

Raman spectroscopy is a powerful light scattering technique used to investigate a very wide range of samples types (organic and inorganic), including biomolecules and microorganisms. This technique is especially attractive because it demonstrates the ability to perform real-time non-invasive (minimally invasive) imaging without the use of biomarkers, its relativity low cost, its portability and the capability of acquired a lot of data in a short time. Samples for conventional Raman spectroscopy measurement can be either in bulk or in microscopic amounts and no special sample preparation required. The technique has been used extensively in recent years for the rapid and accurate identification of clinically relevant microorganisms both remotely and in situ, thus appears to be a convenient technique for the study of microbes. Despite such advantages, the Raman effect is inherently very weak where approximately one in $\sim 10^{5}$ photons are inelastically scattered from a sample. Compared with luminescence-based processes, normal Raman spectroscopy has a small cross-section $\left(\sim 10^{-30} \mathrm{~cm}^{2}\right.$ molecule $\left.{ }^{-1}\right)$ [7], thus precluding the possibility of biomolecules detection at extremely low concentrations and difficult to disentangle the complex overlapping bands from biological macromolecules. Therefore, many Raman based techniques have been developed to deal with the short-coming mentioned above. Surface Enhanced Raman Spectroscopy (SERS), Resonance Raman Spectroscopy (RRS) are a few such techniques that have been 
developed for a wide range of applications, including microbes and cellular detection, in situ monitoring of biological macromolecules at a physiological concentration.

Surface-enhanced Raman spectroscopy (SERS) is a modern spectroscopy technique where a weak Raman signal of a functional group of a Raman -active molecule is greatly enhanced when the molecule is adsorbed on the surface of specially prepared coinage metals, in particular, $\mathrm{Ag}, \mathrm{Au}$, or $\mathrm{Cu}$ [8, 9]. Two different phenomena are responsible for the SERS enhancement: firstly and mostly, the classical nearfield electromagnetic effect and secondly, the chemical effect [10-15], which allows detecting the local composition of biomolecules at extremely low concentrations (down to a single molecule) [16, 17]. Altogether, the SERS technique provides enhancement up to several orders of magnitude if the excitation source equals with the energy of an electronic transition. Signal enhancements as high as $10^{14}$ times over normal Raman scattering have been reported $[16,18]$, which gives SERS spectroscopy a huge potential for various applications where sensitivity is needed in molecular detection. Thanks to the ability to provide direct information on chemical composition, the SERS technique has received tremendous attention in various research fields including biomedical, biological sensors and material science.

Molecular discrimination of different microbes is very important when monitoring changes in cellular structure or during classification of cells and bacteria. SERS, which is becoming popular for the chemical characterization of biological species, is an ideal tool to investigate the class of compounds, which constitutes an intracellular and extracellular environment of microbes, especially bacteria and yeast [19-21]. The detection of yeast and bacteria using Raman and SERS has been reported in several publications [19, 20, 22-29], resulting in a wealth of information about the chemical signatures of different biological components associated with DNA, proteins, lipids and RNA and other biomolecules. However, in most cases, the fingerprint peaks have been difficult to interpret due to extremely low physicochemical concentration and adverse influence of many kinds of background impurities, often encountered in nature, resulting in anomalous peaks (multiple peaks) and distortions. In particular, the interaction between the different biomolecules with their surrounding matrix becomes more complicated in lysate cell, since the concentration may decrease lower than those found in the physiological conditions. Thus, in order to avoid the matrix influence, a careful substrate preparation needs to be adapted before the sample is subjected to a SERS measurement. In order to clearly identify species, some studies have combined Raman with techniques like chemometrics [25].

In this contribution, we report the SERS spectra of yeasts (lysate and non-treated cells) mixed with highly SERS active aggregated Ag nanoparticles. In this method, the SERS spectra associated with the 
molecular features of the yeast cells give rise to intense bands that provide information about the chemical composition of the fungi and enable us to detect molecular (low concentration) differences between yeast species. Furthermore, this enables us to differentiate yeast species based on their minor molecular variation without any additional methods, and also within a complicated matrix. In addition, highresolution images of the mixture of fungus and aggregated Ag nanoparticles are taken with helium ion microscopy (HIM). These images provide the cell morphology and the distribution of the Ag nanoparticles on the surface of the yeast cells, which can be attributed to differences in cell adhesion and further to some changes in the observed spectra. This study suggests that such an approach combining of SERS and HIM may provide a better understanding of the structural complexity of yeast, thus enabling development of powerful detection techniques of these industrially relevant species without using any coupling agents. These results open possibilities for developing sensitive and robust biosensors for controlling the food quality and safety.

\section{Materials and Methods}

\section{$2.1 \quad$ Reagents}

Unless stated otherwise, all chemicals, such as silver nitrate, trisodium citrate, sodium chloride, magnesium chloride, sodium hydroxide, 2-propanol and ethanol, were purchased from Sigma-Aldrich (St. Louis, MO, USA). All the chemicals were of analytical grade and used as received.

\subsection{Experimental setup}

The measurements were made on a Senterra dispersive Raman Microscope (BRUKER, Sweden). Confocal Raman spectroscopy was used to acquire Raman and SERS spectra of bacteria-nanoparticles samples. Raman spectra were obtained using a near-infrared diode laser (785 nm) as an excitation and collecting the Raman scattering in $180^{\circ}$ geometry by a Peltier cooled $\left(-70{ }^{\circ} \mathrm{C}\right)$ charge-coupled device (CCD) camera (255×1024 pixels), focusing only within the fingerprint regions $\left(400-2000 \mathrm{~cm}^{-1}\right)$. For all biological samples, a near-IR (785 or $800 \mathrm{~nm}$ ) laser is a superior light source in order to avoid fluorescence and photodecomposition interference observed with all visible range excitation sources. The spectrometer was equipped with a diffraction grating of 1200 grooves $/ \mathrm{mm}$ and the slit gave a spectral resolution of $2 \mathrm{~cm}^{-1}$. The laser power at the sample ranged from 1 to $10 \mathrm{~mW}$ and the acquisition time ranged from 1 to 5 seconds. Microscope objectives (20, 50 and 100x, NA 0.40, 0.75 and 0.9) were used to focus the laser light on the sample, which consisted of 20 to $50 \mu \mathrm{L}$ drop of mixed sample on a cleaned microscopic slide. The area of the laser spot on the samples was approximately $1 \mu \mathrm{m}$ in diameter (when using 100x objective). The system was calibrated using built-in templates and internal Raman standards. 
Varying amount of yeast cells (non-treated and lysate) mixed with $250 \mu \mathrm{L}$ of aggregated Ag nanoparticles and incubated for $30 \mathrm{~min}$. For the first beer sample, $10 \mu \mathrm{L}$ of beer was applied into a $300 \mu \mathrm{L}$ solution containing Ag nanoparticles and yeast cells, and for the second sample, $10 \mu \mathrm{l}$ of yeast-AgNPsolution was added to $400 \mu \mathrm{L}$ of beer. The mixture was further incubated for another $30 \mathrm{~min}$, and then casted on a clean glass slide for the SERS measurement.

The SERS spectra were collected by mapping a small area on the slide (100 $\mu \mathrm{m} \times 100 \mu \mathrm{m})$. Examples of recorded spectra for each species without any spectral treatments are shown in Supporting Material Figures S2-S4. They indicate high reproducibility of the method. Then the spectra from each collection were averaged and baseline-corrected by rubber band algorithm (using defaults of Origin 8 software). All the measurements were performed in triplicate for each yeast cell.

To determine the morphology of the silver particles, transmission electron microscope (TEM) images were taken on a JEOL-1400 operating at $80 \mathrm{kV}$. The samples were prepared by depositing and drying $5 \mu \mathrm{L}$ of the Ag nano-aggregates on 200 TEM mesh copper grids (FORMVAR Carbon Film). Absorption spectra were recorded from the solution using a UV/Vis spectrometer (Lambda 850, PerkinElmer, Massachusetts, USA). Visual inspection of the nanoparticles is performed on the TEM image to determine the shape and the size of the AgNPs.

The yeast samples were imaged with an Orion NanoFab (Carl Zeiss AG, Germany) helium ion microscope operating at $30 \mathrm{keV}$ accelerating voltage and $0.2-0.4 \mathrm{pA}$ beam current. Charge neutralization with a flood gun allowed imaging non-conductive biological samples without using a conductive metal or carbon film on the sample. Charge neutralization was done after each line scan measured with $0.5 \mu$ s dwell time. Individual line scans were averaged 128 times for the final image. The chamber pressure during the imaging was $1 \cdot 10^{-7}$ mbar, working distance was $9 \mathrm{~mm}$ and the pixel resolution of the images were 2048x2048 pixels. The collected images were adjusted for black and white points to fill the full range of 256 grey levels.

\subsection{Preparation of SERS-active Ag nano-aggregates}

The aggregated silver nanoparticles were synthesized for generating enhanced Raman signals using previously reported methods [30]. All glassware was cleaned in aqua regia (3 parts $\mathrm{HCl}, 1$ part $\mathrm{HNO}_{3}$ ), rinsed with Nano-pure $\mathrm{H}_{2} \mathrm{O}$ (SG Water USA, LLC), and then oven dried prior to use. In a typical procedure, an aqueous solution of $\mathrm{AgNO}_{3}(1 \mathrm{mM}, 200 \mathrm{~mL})$ was brought to reflux while vigorously stirring, and then $4 \mathrm{~mL}$ of a $38.8 \mathrm{mM}$ trisodium citrate solution was quickly added, which resulted in a change from colorless to grayish yellow, indicating that the reaction was completed. After the color change, the solution was refluxed for an additional $60 \mathrm{~min}$, allowed to cool down to room temperature and subsequently stored at $4^{\circ} \mathrm{C}$. The average diameter of the synthesized mostly spherical AgNPs was 
measured using TEM micrographs and its value was found to be $100 \pm 5 \mathrm{~nm}$. After six months of storage, the solution contained an aggregated mixture of spherical, wire and rod-shaped nanoparticles as observed from the obtained TEM images (see example in Figure 1). For a time course study of stability, a small amount of AgNP stock solution was diluted with water and the UV-Vis absorbance measurement recorded every two weeks. A typical UV-VIS spectrum is shown in Figure 1.

\subsection{Preparation of yeast samples}

Saccharomyces cerevisiae (Roberts Ltd. \#229264) was grown in Yeast Malt Broth (YM-Broth) (5 g/l peptone, $3 \mathrm{~g} / \mathrm{l}$ yeast extract, $3 \mathrm{~g} / \mathrm{l}$ malt extract, $10 \mathrm{~g} / \mathrm{l}$ dextrose, $\mathrm{pH} 6.2$; Sigma Y3757) at $30^{\circ} \mathrm{C}$ for $\sim 20$ hours with constant shaking ( $\sim 150 \mathrm{rpm}$ ). The cultures were then centrifuged at $5000 \mathrm{~g}$ for $20 \mathrm{~min}$ at $4{ }^{\circ} \mathrm{C}$, after which the supernatant was discarded and the pellet was suspended in $100 \mathrm{~mL}$ of gold $\mathrm{dH}_{2} \mathrm{O}$. This was still repeated to remove the residual growth media, after which cell pellets were resuspended in 50 $\mathrm{mL}$ of $\mathrm{dH}_{2} \mathrm{O}$ and transferred into smaller containers. To prepare the cellular lysate, the cells resuspended in $\mathrm{dH}_{2} \mathrm{O}$ were treated three times with an Emulsiflex C3 homogenizator (AVESTIN Europe GmbH). All the samples were stored in $0.5 \mathrm{~mL}$ microcentrifuge tubes in $-80^{\circ} \mathrm{C}$ until the analysis.

Dekkera bruxellensis wild yeast strain was purchased from VTT Technical Research Centre of Finland Ltd. (VTT C-00348). It was cultivated in YM-Broth for $\sim 20 \mathrm{~h}$ at $+27{ }^{\circ} \mathrm{C}$ with constant shaking ( $150 \mathrm{rpm}$ ). The culture was then treated as described above for $S$. cerevisiae.

Wickerhamomyces anomalus (former name Pichia anomala) was purchased from VTT Technical Research Centre of Finland Ltd. (VTT C-02470). It was cultivated in YM-Broth for $\sim 20 \mathrm{~h}$ at $+25{ }^{\circ} \mathrm{C}$ with constant shaking ( $150 \mathrm{rpm})$. The culture was then treated as described above for $S$. cerevisiae.

All the yeast cultures are originating from individual colonies picked from agar plate. 


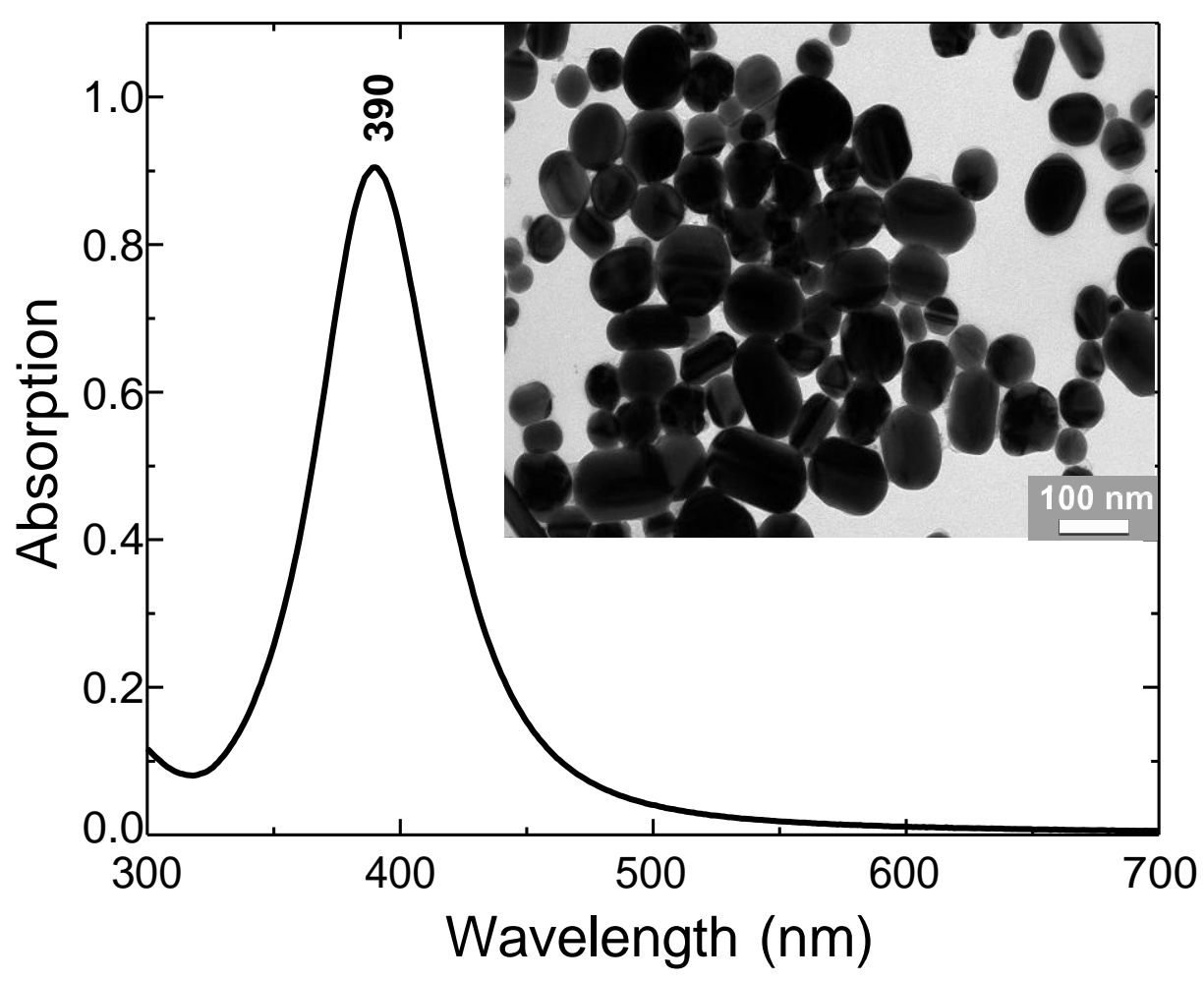

Figure 1. UV-Vis spectrum of aggregated silver nanoparticles. The spectrum shows a clear absorption band at $390 \mathrm{~nm}$. The inset shows a high magnification transmission electron microscope image of a variety of structural features, i.e. hot-spots with plasmonic gap mode forming at the junctions of two or more Ag nanoparticles, that may be responsible for signal enhancement.

\section{Results and Discussion}

\subsection{SERS spectra of yeasts (lysates)}

Typical SERS spectra of the lysates from three yeast species, Saccharomyces cerevisiae, Dekkera bruxellensis, and Wickerhamomyces anomalus (former name Pichia anomala), mixed with aggregated silver nanoparticles are shown in Figure 2a in the range between 400 to $1800 \mathrm{~cm}^{-1}$. The recorded spectra were highly reproducible as demonstrated by the examples of the non-treated data in Supporting Material figures S2-S4. The full list of peak positions along with the appropriate assignments of the three microbes is presented in Table 1.

The SERS spectra of the microbes show complex features dominated by the functional groups of proteins, lipids, and purines. However, in the spectral window between 500-1000 $\mathrm{cm}^{-1}$ (fingerprint area), we noticed that the SERS peak pattern of Wickerhamomyces anomalus is clearly different compared with those from Saccharomyces cerevisiae and Dekkera bruxellensis spectra, as shown in Figure 2a. There is a great variability in both the band shapes and relative intensities of most of the modes and even a new peak 
is observed around $790 \mathrm{~cm}^{-1}$. This can be attributed to the fact that the adsorption behavior of Wickerhamomyces anomalus on Ag nanoaggregate is different than those of Saccharomyces cerevisiae and Dekkera bruxellensis species due to its morphological features (its structural features are found to be different from various groups of fungus) [31]. For this reason, we will discuss briefly the two SERS spectra in more detail in the next paragraph.

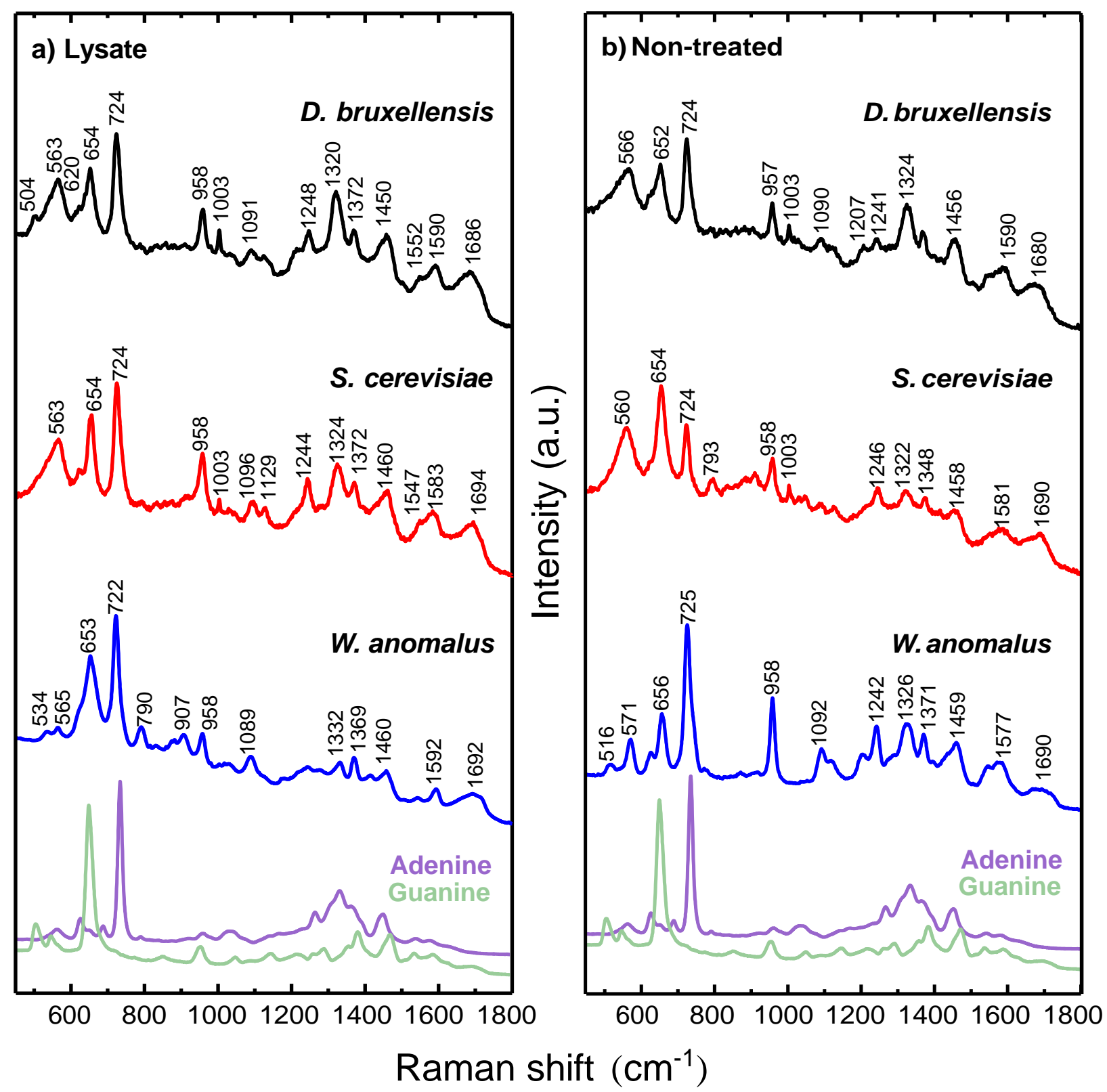

Figure 2. SERS spectra of (a) lysed and (b) non-treated yeast cells: Dekkera bruxellensis, Saccharomyces cerevisiae, and Wickerhamomyces anomalus. For comparison, there are also SERS spectra of Adenine and Guanine measured from aqueous solution (1 mM) mixed with Ag 
nanoparticles. Under the experimental conditions described in the methods, SERS spectra were acquired using 785nm a near-infrared diode laser for excitation. The laser power at the sample was 1 to $10 \mathrm{~mW}$, acquisition time $=1$ to $5 \mathrm{sec}$ and the laser beam passes through 50 and $100 \mathrm{x}$ objective and focuses on the sample. All spectra were baseline corrected by the rubber band algorithm and intensity-normalized.

The SERS spectra of the three microbes exhibit a number of marker modes spreading well over the region between $500-1700 \mathrm{~cm}^{-1}$. However, the three microbes gave similar spectral features, except the obvious minor differences in intensities, positions, and peak sharpness. The major feature (the dominating mode) in all the spectra is the pronounced intense band centered at $724 \mathrm{~cm}^{-1}$ which is assigned to the ring breathing mode of adenine (A) base with some degree of mixing with the other bases as well as purines in general (Figure 2a \& Table 1). The ring breathing mode of the guanine (G) and thymine (T) also appears as a weak band at $1374 \mathrm{~cm}^{-1}$. However, these two modes appear to have slightly shifted, i.e., from 724 to $722 \mathrm{~cm}^{-1}$ and 1372 to $1369 \mathrm{~cm}^{-1}$ in the Wickerhamomyces anomalus spectrum. Such spectral shift can be attributed to the differences between the underlying molecular features of the species. The next intense peak occurs at $654 \mathrm{~cm}^{-1}$, which is also related to the ring breathing mode in $\mathrm{G}$ and T. In addition, there is a weak shoulder peak observed at $620 \mathrm{~cm}^{-1}$ which originate from the C-C twisted mode of phenylalanine (Figure 2a, Table 1). However, the later band is not well resolved in all three spectra but discernible. It is also worth noting, however, that the SERS spectra obtained from the molecular layers coating the metal surface and the relative intensity of the molecular components are modulated by the orientation of the adsorbate on the surface of the aggregated metal. This phenomenon is manifested over the entire SERS spectra. Moskovits described the selection rule of molecules absorbed (orientation) onto metal surfaces in his famous publication [32]. For the sake of comparison, the powder spectra of A and G are shown in Figure 2.

Table 1. SERS mode assignments of Saccharomyces cerevisiae, Dekkera bruxellensis and Wickerhamomyces anomalus in the lysate and non-treated forms.

\begin{tabular}{|c|c|c|c|c|c|c|}
\hline \multicolumn{2}{|c|}{$\begin{array}{c}\text { Saccharomyces } \\
\text { cerevisiae } \\
\left(\mathrm{cm}^{-1}\right)\end{array}$} & \multicolumn{2}{|c|}{$\begin{array}{c}\text { Dekkera } \\
\text { bruxellensis } \\
\left(\mathrm{cm}^{-1}\right)\end{array}$} & \multicolumn{2}{|c|}{$\begin{array}{c}\text { Wickerhamomyces } \\
\text { anomalus } \\
\left(\mathrm{cm}^{-1}\right)\end{array}$} & \multirow[t]{2}{*}{ Tentative assignments $*$} \\
\hline lysate & $\begin{array}{c}\text { non- } \\
\text { treated }\end{array}$ & lysate & $\begin{array}{l}\text { non- } \\
\text { treated }\end{array}$ & lysate & $\begin{array}{c}\text { non- } \\
\text { treated }\end{array}$ & \\
\hline 508 & 516 & 504 & & & 515 & $\begin{array}{c}v(\mathrm{~S}-\mathrm{S}) \text { disulfide (amino acid } \\
\text { cysteine) }\end{array}$ \\
\hline 563 & 559 & 564 & 563 & 565 & 571 & $\mathrm{~T} / \mathrm{C}, \mathrm{G}$ \\
\hline
\end{tabular}




\begin{tabular}{|c|c|c|c|c|c|c|}
\hline 602 & & & & & 616 & C-C twisting mode of Phe \\
\hline 627 & 621 & 626 & 627 & 626 & 627 & C-C twisting mode of Phe \\
\hline 654 & 654 & 652 & & 653 & 656 & G, T (ring-breathing modes) \\
\hline 724 & 723 & 724 & 724 & 722 & 725 & $\begin{array}{c}\text { Adenine } \\
\text { (ring breathing modes of } \\
\text { purines) }\end{array}$ \\
\hline \multirow[t]{2}{*}{798} & 794 & & & 791 & & $v(\mathrm{O}-\mathrm{P}-\mathrm{O}) \mathrm{RNA}$ \\
\hline & & & & 815 & & $v(\mathrm{O}-\mathrm{P}-\mathrm{O}) \mathrm{RNA}$ \\
\hline 831 & 834 & & & 831 & & $v_{\mathrm{s}}(\mathrm{O}-\mathrm{P}-\mathrm{O})$ str., $\mathrm{T}$ \\
\hline \multirow[t]{2}{*}{856} & & & & 859 & & Tyrosine \\
\hline & & & & 874 & 870 & Polysaccharides \\
\hline 913 & 883 & & & 882 & & Phosphodiester, Deoxyribose \\
\hline 920 & 909 & & & 907 & & Skeletal C-C, $\alpha$-helix \\
\hline \multirow[t]{2}{*}{957} & 959 & 959 & 957 & 958 & 957 & $v(\mathrm{C}-\mathrm{O})$ and $(\mathrm{C}-\mathrm{N})$ of proteins \\
\hline & & 981 & 980 & 981 & & $v(\mathrm{C}-\mathrm{C})$ of proteins \\
\hline \multirow[t]{2}{*}{1003} & 1003 & 1003 & 1003 & 1003 & 1003 & Phe \\
\hline & & & 1017 & 1017 & & $\delta(\mathrm{C}-\mathrm{H})$ of $\mathrm{T}$ \\
\hline 1038 & 1030 & 1027 & 1028 & 1023 & 1026 & $\begin{array}{c}\delta(\mathrm{CH}) \text {, Phe (protein } \\
\text { assignment) }\end{array}$ \\
\hline 1092 & 1089 & 1091 & 1092 & 1089 & 1092 & $\mathrm{PO}_{2}^{-}$in nucleic acid \\
\hline \multirow[t]{2}{*}{1127} & 1126 & 1124 & & & 1119 & $\begin{array}{c}\delta(\mathrm{C}-\mathrm{H}), \text { Phe (protein } \\
\text { assignment) }\end{array}$ \\
\hline & & & 1172 & 1172 & & $\delta(\mathrm{C}-\mathrm{H})$ of tyrosine and proteins \\
\hline \multirow[t]{2}{*}{1203} & 1204 & 1211 & 1205 & 1198 & 1205 & Amide III, $v_{\text {as }}\left(\mathrm{PO}_{2}^{-}\right)$str. \\
\hline & & & 1239 & 1234 & & Amide III \\
\hline \multirow[t]{2}{*}{1244} & 1245 & 1245 & 1243 & 1242 & 1241 & Amide III, $v_{\text {as }}\left(\mathrm{PO}_{2}^{-}\right)$str. \\
\hline & & & & & 1289 & $\mathrm{C}$ \\
\hline 1324 & 1322 & 1325 & 1324 & 1332 & 1326 & $\mathrm{G}, \mathrm{CH}_{3} \mathrm{CH}_{2}$ deformation \\
\hline \multirow[t]{3}{*}{1372} & 1374 & 1372 & 1368 & 1370 & 1371 & $\begin{array}{l}\text { T,A,G (ring breathing modes } \\
\text { of purines and pyrimidines) }\end{array}$ \\
\hline & & & & & 1395 & $\rho(\mathrm{CH})$ \\
\hline & & & & 1414 & & $\mathrm{CH}_{2}$ sciss (lipids), G,A \\
\hline 1458 & 1452 & 1461 & 1456 & 1458 & 1460 & Nucleic acid modes \\
\hline 1550 & & 1551 & & 1548 & & Amide II \\
\hline
\end{tabular}




\begin{tabular}{|c|c|c|c|c|c|c|}
\hline 1583 & 1581 & 1589 & 1589 & 1576 & 1579 & $\begin{array}{c}\text { C=C bending mode of } \\
\text { phenylalanine }\end{array}$ \\
\hline 1693 & 1686 & 1687 & 1672 & 1692 & 1686 & Amide I of protein \\
\hline
\end{tabular}

*All the assignments are from references 12, 16,18 \& 31

${ }^{\mathrm{e}} v$, stretching; $\delta$, bending; $\rho$, rocking; $\tau$, torsion; wagg, wagging; twist, twisting; sciss, scissoring; def, deformation; as, asymmetric; s, symmetric; C, cytosine; T, thymine; A, adenine; G, guanine; Phe, phenylalanine; Tyr, tyrosine

Furthermore, a broad peak was observed at $563 \mathrm{~cm}^{-1}$. The characteristic broad and weak bands observed at 563 and $534 \mathrm{~cm}^{-1}$ can be assigned to the T/cytosine (C) and G with some degree of mixing from the C-C twisting mode of Phe (Table 1 and Figure 2a). Interestingly, the peak at $536 \mathrm{~cm}^{-1}$ is extremely weak in Wickerhamomyces anomalus cell sample compared with those from Saccharomyces cerevisiae and Dekkera bruxellensis species, as shown in Figure 2a. Apart from these characteristic peaks, the last one observed at $504 \mathrm{~cm}^{-1}$ is associated with the disulfide (S-S) modes of the cysteine residue in a protein. This mode is clearly indicating that the proteins in the two species contain a plenty of disulfide bonds. However, the absence of this mode in $W$. anomalus species may be ascribed with the low concentration of proteins with disulfide bonds.

The third strongest signal in the SERS spectra of $S$. cerevisiae and D. bruxellensis (Figure 2a) appears at $958 \mathrm{~cm}^{-1}$ assigned to the stretching mode (C-O), admixed with $v(\mathrm{C}-\mathrm{N})$ of proteins. However, this mode is relatively weak and broad in $W$. anomalus species compared with the two spectra. The different relative intensities of the peaks observed may give some indication of the relative abundances of each component present in each species. Besides this band, in W. anomalus we also observe a peak at 790 $\mathrm{cm}^{-1}$ which is largely absent in the other two species. In addition to this mode, there are two additional peaks at $907 \mathrm{~cm}^{-1}$ attributed to the C-O-C skeletal mode and the weak shoulder at $875 \mathrm{~cm}^{-1}$ which is assigned to $v(\mathrm{C}-\mathrm{C})$, respectively. These species-specific modes could serve as a marker to differentiate species of micro-organism owing to the structural uniqueness of the yeast species. Furthermore, it can be seen in Figure 2a that the region between 910 to $1700 \mathrm{~cm}^{-1}$ contains a complex shape of bands (low intensities) and suffer from a lack of high signal-to-noise ratios indicating the presence of several overlapping peaks. This is due to the fact that the cell contains several macromolecules and these constituents co-adsorb on the metal surface, resulting in peak broadening and distortion. In addition, the relative intensity of the molecular components is modulated by the orientation of the adsorbate on the hot spots formed between closely spaced Ag nanoparticles due to a high-intensity plasmonic gap mode. These spectral regions will be analyzed in detail in order to discriminate the three species based on their spectral characterstics. 
The next medium-intense band is observed at $1324 \mathrm{~cm}^{-1}$ which correspond to guanine and $\mathrm{CH}_{3} \mathrm{CH}_{2}$ deformation. Another weak band appearing at $1372 \mathrm{~cm}^{-1}$ can be assignable to ring breathing mode of thymine, adenine and guanine (Table 1). These two bands are too weak and broad to be observed in the $W$. anomalus spectrum, indicating the presence of an overlapping band. The C-C skeletal stretching vibration of phenylalanine ring together with $\mathrm{C}-\mathrm{H}$ in-plane phenylalanine yields the bands at 1003 and $1030 \mathrm{~cm}^{-1}$. However, this band $\left(1003 \mathrm{~cm}^{-1}\right)$ is diminishing in intensity and shifts to $978 \mathrm{~cm}^{-1}$ in $W$. anomalus sample, indicating that the matrix affects the phenylalanine environment. The intensity of the band at $1030 \mathrm{~cm}^{-1}$ also decrease significantly in $W$. anomalus cell. The complex symmetrical stretching mode of $\mathrm{PO}_{2}$ is composed of C-N, C-O, and $v(\mathrm{C}-\mathrm{C})$ skeletal of acyl backbone in lipid observed at 1096 and $1129 \mathrm{~cm}^{-1}$ in S. cerevisiae and D. bruxellensis samples and show a minor spectral shift to 1089 and $1126 \mathrm{~cm}^{-1}$ in W. anomalus spectrum.

The amide I, II and III vibrational modes are conformational sensitive and exhibits extremely complex features involving the coupling of other vibrational modes. The amide group involves primarily $\mathrm{C}=\mathrm{O}$ stretching, in-plane modes of $\mathrm{N}-\mathrm{H}$ bending, and $v(\mathrm{C}-\mathrm{C})$ vibrational modes. In the region between 1100 to $1700 \mathrm{~cm}^{-1}$, very strong amide III mode for $\alpha$-sheet disordered conformation is observed at 1245 $\mathrm{cm}^{-1}$ for S. cerevisiae, at $1248 \mathrm{~cm}^{-1}$ for DB and very weak band for $W$. anomalus which lies at $1241 \mathrm{~cm}^{-1}$. The presence of a shoulder at $1220 \mathrm{~cm}^{-1}$ is also due to amide III and arising from the coupling of C-N stretching and N-H bonding. The amide I SERS band is observed at 1694 (SC), 1686 (D. bruxellensis) and $1692 \mathrm{~cm}^{-1}$ in $W$. anomalus species. This band is primarily arising due to $\mathrm{C}=\mathrm{O}$ stretching mode coupled with the in-plane N-H bending and C-N stretching bands. The high wavenumber observed may be associated with the type of the secondary structure dominating in these species. Thus, the observation of the high wavenumber peaks provides evidence for the existence of $\beta$-pleated sheet configuration in all the cells. [33] This result is further confirmed by the observation of the amide III bands at $1245 \mathrm{~cm}^{-1}$.

\subsection{SERS spectra of yeasts (non-treated cells)}

The vibrational spectra of the S. cerevisiae, D. bruxellensis, and W. anomalus in non-treated form are dominated by proteins, lipids, and purine groups. Figure 2b shows the SERS spectra collected from each cell of the three species absorbed on the aggregated silver particles (Figure 3). As seen in Figure 2b, significant spectral shifts and relative intensity changes are observed when compared with the cells in the lysate form (Figure 2a). We will discuss this rather clear difference between the two forms in detail in the following section. Moreover, the high-resolution HIM images are used to analyze the formation of the aggregates around the fungi cells (Figure 3).

In the region between 500 to $1200 \mathrm{~cm}^{-1}$ shown in Figure 2b, the SERS spectra taken from the nontreated cells is characterized by medium/high-intensity modes observed at 566, 652, 724, 957, 1003 and 
$1092 \mathrm{~cm}^{-1}$ (as already assigned in Figure 2a) attributed to disulfide bond, adenine ring breathing, vs $\left(\mathrm{CH}_{3}\right)$ of protein ( $\alpha$-helix), C-C skeletal of phenylalanine (Phe) and symmetric stretching mode of $\mathrm{PO}_{2}$ group (Table 1). These bands show a change in peak intensities and a minor spectral change. The peak intensities at 724 and $958 \mathrm{~cm}^{-1}$ increase without a shift in $W$. anomalus, and simultaneously the peak at $654 \mathrm{~cm}^{-1}$ is intensified in $S$. cerevisiae species compared with the other two species, which indicated the adsorption of the purines on the hot-spot. Similarly, the broad peak at $566 \mathrm{~cm}^{-1}$ shifts to $560 \mathrm{~cm}^{-1}$ in $S$. cerevisiae, while it exhibits sharp peak and shifts to $571 \mathrm{~cm}^{-1}$ in $W$. anomalus cell. In addition, a peak at $516 \mathrm{~cm}^{-1}$ was observed in the same spectrum (W. anomalus). This mode is assigned to S-S stretching mode within the protein. Lastly, the last two bands observed at 1003 and $1092 \mathrm{~cm}^{-1}$ assigned to C-H inplane phenylalanine (proteins) and symmetric $\left(\mathrm{PO}_{2}^{-}\right)$are very weak for the two species $(D$. bruxellensis and S. cerevisiae). The former band is extremely weak in $W$. anomalus, while the $1092 \mathrm{~cm}^{-1}$ mode increases in intensity. However, a strong mix with $1116 \mathrm{~cm}^{-1}$ mode also observed. A similar observation was made in the SERS spectra of the lysate form in $W$. anomalus cells. The selected SERS assignments are presented in Table 1.

The relatively high amount of the purine (adenine, guanine) signals in the SERS spectra of the nontreated cells is a bit surprising, as one would expect more cell wall associated signals arising from the intact cells. However, previous studies revealed that these peaks are commonly detected in SERS experiments carried out for intact yeast and bacterial cells [22, 34-36]. It is possible, that those signals are associated with degradation of nucleic acids and nucleotides due to a cell starvation, which leads into release of the adenine and guanine outside the cells, as described recently for starved bacterial cells by Premasiri et al. [35]. Also, traces of nucleic acids could be released from cells broken during the preparation, but we did not observe significant DNA release in the sample preparation. In addition, it is also possible that some of the AgNPs have been able to penetrate into cells and thus yield SERS signal from the purines inside the cells. These particles would not be visible in the HIM images either, since HIM is very surface sensitive and it does not allow detection of the nanoparticles through the cell wall.

The complex shape of the modes between 1200 to $1700 \mathrm{~cm}^{-1}$ is due to the presence of several overlapping modes in phosphate and amide groups of proteins and the ring of purines DNA/RNA. We noticed that the amide III band at $1241 \mathrm{~cm}^{-1}$ is very weak in both $D$. bruxellensis and S. cerevisiae spectra, whereas it exhibits a medium and sharp peak at $1242 \mathrm{~cm}^{-1}$ in $W$. anomalus cell. This may indicate a different orientation of the amide II component on the surface of the Ag-aggregates, which is consistent with the fact that the $W$. anomalus species is very different compared with that $D$. bruxellensis and S. cerevisiae. In addition, a weak shoulder appears at $1207 \mathrm{~cm}^{-1}$ and its intensity become lower in $S$. cerevisiae, while increases in W. anomalus spectrum. 
In the amide I region (1590 and $1680 \mathrm{~cm}^{-1}$ ), the modes observed at 1456, 1590 and $1680 \mathrm{~cm}^{-1}$ are broader (much weaker) and the spectra suffer from a low signal/noise ratio, indicating a very complex spectral pattern due to, the most probable, substancial amoumt of spectral mixing. For example, the 1680 $\mathrm{cm}^{-1}$ (D. bruxellensis) shifts to $1690 \mathrm{~cm}^{-1}$ (S. cerevisiae and W. anomalus), thus providing evidence to some overlapping with other modes. We also identify a weaker shoulder band around $1547 \mathrm{~cm}^{-1}$ which is possibly due to amide II as suggested in the literature [20]; this band indicates the presence of parallel $\beta$ sheet structure in all three species. Besides the weak amide II band, another band is observed at $1590 \mathrm{~cm}^{-1}$ , which is considered to be due to $\mathrm{C}=\mathrm{C}$ bending of Phy and a minor mixing from amide $\mathrm{I}$. This mode is strongly shifted to lower wavenumber $1581 \mathrm{~cm}^{-1}$ (S. cerevisiae) and $1577 \mathrm{~cm}^{-1}$ (W. anomalus), which indicated strong mixing with other modes.

\subsection{Statistical Analysis}

The closeness and variability in the SERS spectra of the three yeasts samples can be attributed to the sample matrix, variability in yeasts composition and the effect of Ag NP aggregation that the individual biomolecules oriented within the hot spots. This is assessed using statistical analysis where standard deviation (SD) is calculated on the respective SERS bands appears in all three samples in respect to their spectral shift. It can be seen in Table $1 \mathrm{~S}$ and $2 \mathrm{~S}$ that the SD of all the SERS bands in both states deviates from the mean value except for the band at $1003 \mathrm{~cm}^{-1}$. For instance, Amide I \&III bands (SD =3.2, 6.6), $\mathrm{CH}_{3} \mathrm{CH}_{2}$ (def) (4.4) and $\mathrm{C}=\mathrm{C}$ (6.5) bending modes of phenylalanine in lysate state are significantly deviate from their mean values and suggesting that the individual modes are different from each other. Similar trends are also observed with the non-treated species such as the band for $v(S-S)$ (6.1), T/C, G (3.5), T,A,G (ring-breathing modes of purines and pyrimidines) (3.0), $\mathrm{C}=\mathrm{C}$ bending mode of phenylalanine (5.3) and Amide I of protein (8.1) show significant matrix and compositional dependency between the three species. 

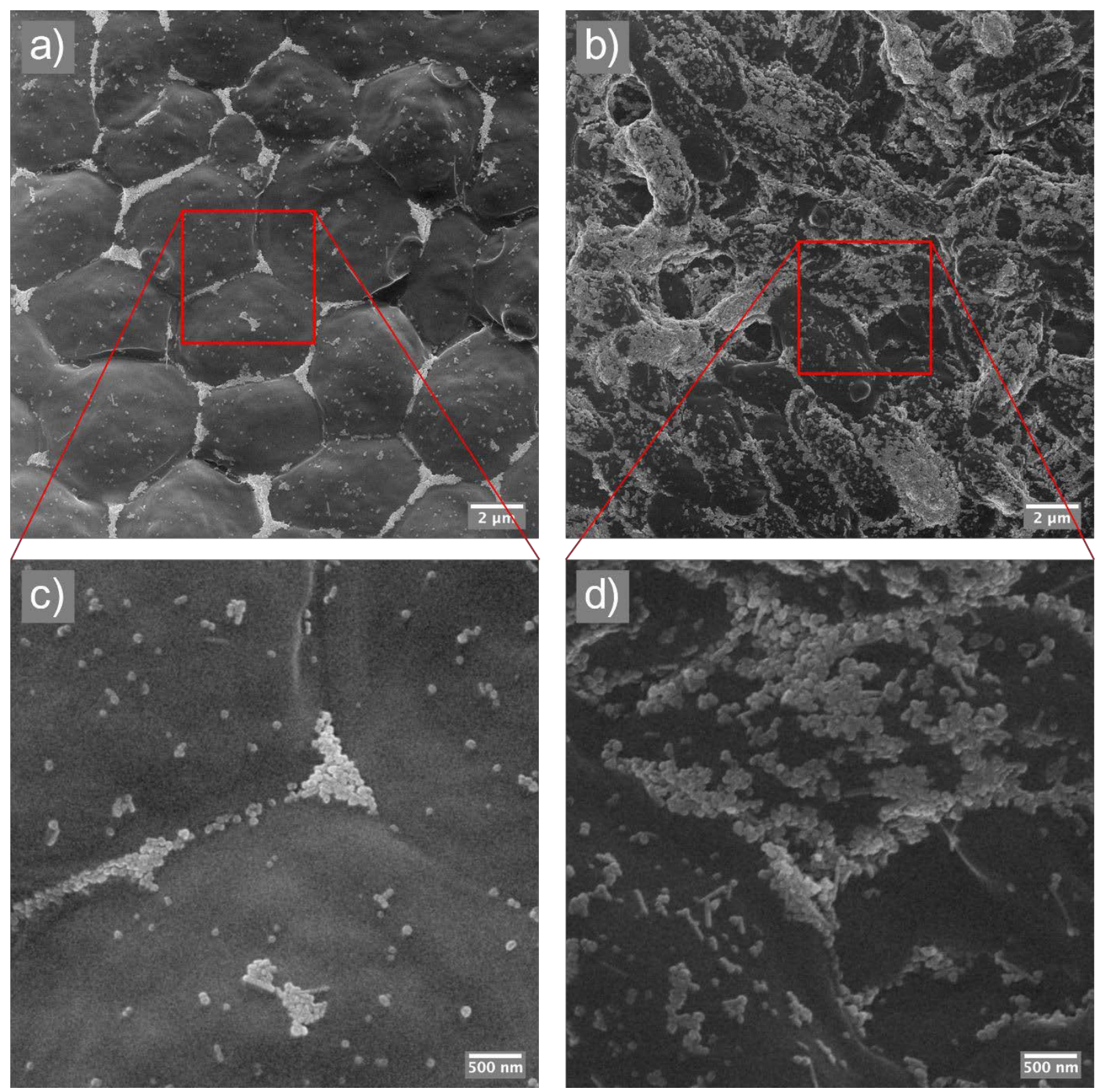

Figure 3. HIM images showing the nano-aggregated silver on yeast cells (non-treated): (a/c) Saccharomyces cerevisiae (b/d) Dekkera bruxellensis. The image size of (a) and (b) is 20x20 $\mu \mathrm{m}^{2}$, and (c) and (d) is $5 \times 5 \mathrm{~m}^{2}$.

\subsection{HIM imaging of the yeasts}

High resolution HIM images of the yeast cells after incubated with the nano-aggregated Ag particles are shown in Figure 3. The images of different yeast species are well resolved allowing the identification of single cells, cell morphology and the way the nanoparticles are distributed around the cells. Each cell type shows a considerable different morphology and selective attachment of the nanoparticles, which is manifested in their SERS spectra. The surface of D. bruxellensis is entrapped in the aggregate (complete coverage), whereas the $S$. cerevisiae is only partially covered by nanoparticles which suggests that the 
interaction between the cell surface with nanoparticles is mostly governed by the morphology and the adherent behavior of the cells (Figure 3). Despite that the SERS spectra of all the cells show a similar signal-to-noise ratio which is sufficient to detect the very weak modes associated with the microcomponents of the cell. This suggests that the NP strongly adsorb onto the surface of the cell or penetrate into the inner cell structure possibly taken up by the cell through endocytosis [37], leading to the agglomeration of the nanoparticles inside the cell. Such phenomenon would lead to the synergetic effect of the inside and outside aggregation of nanoparticles which may form many hot-spots created by Ag-Ag junction where the cell components could be confined and exposed to extremely high local electromagentic field and therefore generate high SERS signal.

Finally, it is worth noting that the SERS spectra of the non-treated cells suffer from the low spectral resolution in all species compared with the spectra collected from lysed cells. This can be rationalized by the constrains of the cell components by the cell wall, resulting in that the macromolecules do not come in a direct contact with the hot-spots of the aggregated Ag particles where the enhancement of scattering occurs. Figure 3 provides some insights into this question and reveals the size mismatch between yeast cells and silver nanoparticles.

\subsection{SERS detection of yeasts (non-treated cells) from beer}

We also investigated the SERS spectra of the three yeast species suspended in beer and thus demonstrated the potential and the high sensitivity of this technique in a complex matrix. Figure 4 shows the SERS spectra of the three types of cells incubated with the silver nanoparticles and then mixed into beer. The observed SERS spectra show a good signal-to-noise ratio compared with those observed for Ag solution without the beer. Similar to the pure SERS spectra, the SERS spectra of the cells in beer is characterized by proteins, lipids, purines and show a similar level of enhancement as those shown in Figure 3. We nevertheless noticed that the SERS spectra of $D$. bruxellensis suffer from a low signal-tonoise ratio compared to the other two SERS spectra (Figure 4). Besides a clear indication of the spectral noise, some bands show an increase/decrease a relative intensity and a spectral shift is observed in peak positions as compared with the pure cells. For instance, the relative intensities of the two bands at 654 and $728 \mathrm{~cm}^{-1}$ were switched and showed a minor spectral shift (2 to $4 \mathrm{~cm}^{-1}$ ). This behavior is probably attributed to the surface properties of the cell suspended in distilled water vs. cell suspended in beer, which provides chemically less demanding condition for the yeast cell. This may lead to changes in accessibility of cellular components for the nanoparticles. Further, the intensity of the previously assigned band centered at the $1241 \mathrm{~cm}^{-1}$ increased and shifted to higher energy mode $\left(1250 \mathrm{~cm}^{-1}\right)$, which means that more of the $\left(\mathrm{PO}_{2}{ }^{-}\right)$moiety may be trapped in the hot-spot in beer-suspended samples. However, the 
remaining modes associated principally with nucleic acid, amide I and phenylalanine show no significant spectral changes.

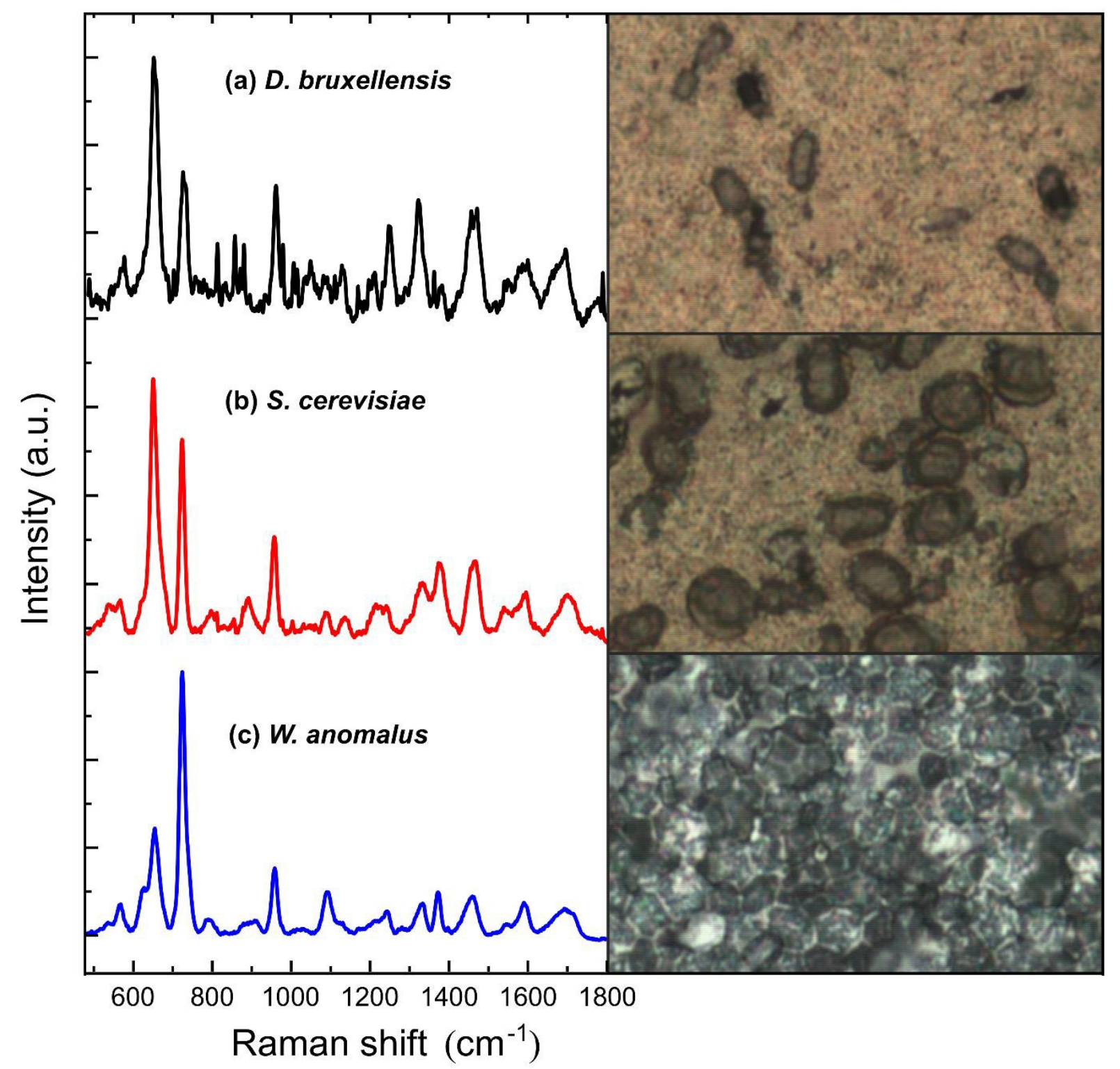

Figure 4. SERS spectra of (a) D. bruxellensis, (b) S. cerevisiae and (c) W. anomalus in beer. The corresponding white light images collected using a microscope are shown. Experimental conditions: Laser power $=1$ to $10 \mathrm{mw}$, acquisition time $=1$ to $5 \mathrm{sec}$ and the laser beam passes through 50 and 100x objective and focuses on the sample.

As with the SERS spectra of $S$. cerevisiae, no significant spectral changes (all modes stay within 1 to $3 \mathrm{~cm}^{-1}$ ) were observed, except in the $1200-1500 \mathrm{~cm}^{-1}$ spectral region, which revealed increased intensity. As already mentioned above, the enhancement of the SERS intensities depends on the orientation of these modes in the junction. Apart from that, the remaining modes are identical with those of the pure SERS 
spectra. Lastly, we have not observed any difference (a major spectral shift or intensities) between the SERS spectra of $W$. anomalus suspended in water or in beer.

To explore the robustness of the technique, we further performed experiments by diluting the three yeast cells in the beer matrix in a higher dilution of 1:40. The observed spectra of the three species still clearly show all the important features of the cells similar to the one seen in Fig. 2 \& 4., though the spectra suffer from a low signal-to-ratios, as expected taken into consideration the dilution factor. The spectra are shown in Supporting Material Figure S1. As a control experiment we also mixed bare AgNPs with beer. Expectedly, the obtained spectra showed no features of the yeasts, as depicted in the Supporting Material Figure S5. Thus the result reaffirms the robustness of the SERS techniqe by being able to discern detection and identification of species regardless of a high background due to the complex chemical environment.

\section{Conclusions}

We used highly SERS-active aggregated silver nanoparticles substrate to discriminate between the three yeast species as lysates and in non-treated cell samples. The SERS spectra of S. cerevisiae and D. bruxellensis were almost identical except a slight spectral shift and change in relative intensities of the phosphate group in DNA/RNA and amide III, implying similar molecular compositions present in these microbes. However, some part of the observed differences in spectra and band intensities may also be attributable to the differences in the accessibility of the chemical structures of the yeasts and in the characteristics of the interactions between yeast molecules and the metal particle surface.

Importantly, the spectral pattern of the lysate and non-treated cell sample show remarkable differences. While cellular components are well accessible in lysate cell, the constricted cell morphology of the non-treated cells leads to relatively weak SERS spectra. This may be due to steric constrains and a macromolecular crowding, preventing access to the hot-spots within the metal particle aggregates (see Figure 3). Additionally, we observed significant spectral differences in the spectrum of Wickerhamomyces anomalus as compared with Saccharomyces cerevisiae and Dekkera bruxellensis, reflecting the obvious molecular composition diversity within the yeast species. Beside a clear indication of the decrease/increase of relative intensities, most of the SERS modes in $W$. anomalus show a shift in peak position to higher/lower wavenumber compared with the $S$. cerevisiae and D. bruxellensis spectra, especially in 1200-1700 $\mathrm{cm}^{-1}$ region. Moreover, it is worth noting that all the three amide groups (I, II \& III) were detected in the SERS spectra present in the species and the broader peaks with high 
wavenumbers $1680 \mathrm{~cm}^{-1}$ (D. bruxellensis), $1690 \mathrm{~cm}^{-1}$ (S. cerevisiae) and $1690 \mathrm{~cm}^{-1}$ (W. anomalus) indicate the presence of parallel $\beta$-sheet structure accessible on the surface of non-treated cells. We also collected the SERS spectra of the same cells in beer medium and analyzed the spectral shift and intensity changes by comparing to spectra obtained in water-suspended cells. The results indicate the extreme sensitivity of the SERS technique that is capable of simultaneously detecting and distinguishing yeast species in a complex media with reasonably high signal-to-noise ratio and spectral reproducibility.

In conclusion, SERS provides a unique platform to detect specific biochemical fingerprints with high sensitivity and specificity at high spatial resolution, which makes the technique suitable for complex mixtures of microbial cultures, and opens possibilities for the development of robust biosensors for monitoring food quality and safety.

Funding: This research was funded by Tekes (project \#1185/31/2013). Academy of Finland is acknowledged for funding for VPH (136288) and JJT (263526). This work also supported by grants from FAPESP (2016/06424-5, 2013/14262-7).

Acknowledgments: Support from BioNavis, Fimlab Laboratories, PBL Brewing Laboratory (VTT) and Orion Diagnostica are gratefully acknowledged. Ulla Kiiskinen (U. Tampere) is acknowledged for technical support in sample preparation. Biocenter Finland is acknowledged for infrastructure support.

Conflicts of Interest: The authors declare no conflict of interest. 


\section{References}

[1] E. Nevoigt, Progress in metabolic engineering of Saccharomyces cerevisiae, Microbiol Mol Biol Rev, 72 (2008) 379-412.

[2] L.V. McFarland, P. Bernasconi, Saccharomyces boulardii. A Review of an Innovative Biotherapeutic Agent, Microbial Ecology in Health and Disease 6(1993) 157-171.

[3] G. Naja, P. Bouvrette, S. Hrapovic, J.H.T. Luong, Raman-based detection of bacteria using silver nanoparticles conjugated with antibodies, Analyst 132 (2007) 679-686.

[4] Fleet.G.H., Cell walls., in: A.H.a.H. Rose, S. (Ed.) The Yeasts, Academic Press, London, London, 1991, pp. 199-277.

[5] F.M. Klis, Cell wall assembly in yeast, Yeast, 10 (1994) 851-869.

[6] P. Orlean, H. Ammer, M. Watzele, W. Tanner, Synthesis of an O-glycosylated cell surface protein induced in yeast by alpha factor, Proc Natl Acad Sci U S A., 17 (1986) 6263-6266.

[7] H.W. Schrotter, H.W. Klockner, Raman scattering cross sections in gases and liquids, in: A. Weber

(Ed.) Raman Spectroscopy of Gases and Liquids, Springer-Verlag, Berlin, 1979, pp. 123-166.

[8] S. Bhavya, R.F. Renee, H. Anne-Isabelle, R. Emilie , P.V.D. Richard SERS: Materials, applications, and the future, Materials, 15 (2012) 16-25.

[9] T. Zhong-Qun, Y. Zhi-Lin, R. Bin, W. De-Yin, SERS From Transition Metals and Excited by

Ultraviolet Light, in: K. Katrin, K. Harald, M. Martin (Eds.) Surface-Enhanced Raman Scattering Physics and Applications, Springer-Verlag Berlin Heidelberg Germany, 2006, pp. 125-147.

[10] M. Moskovits, Surface-enhanced spectroscopy, Rev. Mod. Phys, 57 (1985) 783-826.

[11] G.C. Schatz, Theoretical-studies of surface enhanced Raman-scattering, Acc. Chem. Res., 17 (1984) 370-376.

[12] M. Kerker, Electromagnetic model for surface-enhanced Raman-scattering (SERS) on metal colloids, Acc. Chem. Res., 17 (1984) 271-277.

[13] A. Otto, I. Mrozek, H. Grabhorn, W. and Akemann, Surface-enhanced Raman-scattering, J. Phys.Condens. Matter, 4 (1992) 1143-1212.

[14] P. Kambhampati, C.M. Child, M.C. Foster, A. Campion, On the chemical mechanism of surface enhanced Raman scattering: experiment and theory, J. Chem. Phys., 108 (1998) 5013.

[15] A. Campion, P. Kambhampati, Surface-Enhanced Raman Scattering Chem. Soc. Rev. , 27 (1998) 241- 250.

[16] K. Katrin, W. Yang, Harald Kneipp, L.T. Perelman., I. Irving, R.D. Ramachandra, S.F. Michael, Single Molecule Detection Using Surface-Enhanced Raman Scattering (SERS), Phys. Rev. Lett. , 78 (1997) 1667.

[17] C.J. Constantino, T. Lemma, P.A. Antunes, R. Aroca, Single-molecule detection using surfaceenhanced resonance Raman scattering and Langmuir-Blodgett monolayers., Anal Chem. , 73 (2001) 3674-3678.

[18] K. Katrin, K. Harald, D. Geurt, I. Irving, R.D. Ramachandra, S.F. Michael, Single-Molecule Detection of a Cyanine Dye in Silver Colloidal Solution Using Near-Infrared Surface-Enhanced Raman Scattering Applied Spectroscopy 52 (1998) 175-178.

[19] A. Sujith, T. Itoh, H. Abe, K. Yoshida, M.S. Kiran, V. Biju, M. Ishikawa, Imaging the cell wall of living single yeast cells using surface-enhanced Raman spectroscopy, Anal Bioanal Chem., 394 (2009) 1803-1809.

[20] L. Tibebe, S. Alex, H. Ville, P.H. Vesa, J.J. Toppari., SERS detection of cell surface and intracellular components of microorganisms using nano-aggregated Ag substrate, Vibrational Spectroscopy 83 (2016) 36-45.

[21] D.l.R. José Maria, P.M. Martin-Sanchez., S.-C. Santiago, B. Hermosin., K. Heike, S.-J. Cesareo, Structure of melanins from the fungi Ochroconis lascauxensis and Ochroconis anomala contaminating rock art in the Lascaux Cave, Sci Rep, 7 (2017) 13441.

[22] I. Sayin, M. Kahraman, F. Sahin, D. Yurdakul, M. Culha, Characterization of yeast species using surface-enhanced Raman scattering, Appl Spectrosc, 63 (2009) 1276-1282. 
[23] L. Boyan, M.S.S. Narayana, H.R. Bryan, G.R. Alan, Using surface-enhanced Raman scattering (SERS) and fluorescence spectroscopy for screening yeast extracts, a complex component of cell culture media, Journal of Raman Spectroscopy 43 (2012) 1074-1082

[24] A. Sujith, T. Itoh, H. Abe, A.A. Anas, K. Yoshida, V. Biju, M. Ishikawa, Surface enhanced Raman scattering analyses of individual silver nanoaggregates on living single yeast cell wall, Applied Physics Letters, 92 (2008).

[25] P. Marek, SERS Investigations of Cells, Viruses and Microorganisms, in: G. Elias (Ed.) SurfaceEnhanced Raman Spectroscopy: Bioanalytical, Biomolecular and Medical Applications, Springer International Publishing Switzerland, 2016, pp. 127-148.

[26] S.B. Rodriguez, M.A. Thornton., R.J. Thornton., Raman Spectroscopy and Chemometrics for Identification and Strain Discrimination of the Wine Spoilage Yeasts Saccharomyces cerevisiae, Zygosaccharomyces bailii, and Brettanomyces bruxellensis, Appl Environ Microbiol 79 (2013) 62646270.

[27] C. Wei, M. Li, X. Zhao, Surface-Enhanced Raman Scattering (SERS) With Silver Nano Substrates Synthesized by Microwave for Rapid Detection of Foodborne Pathogens, Front Microbiol, 9 (2018) 2857. [28] W. Ruben, P. Márton, W. Michael, N. Reinhard, E. Martin, S. Michael, P.I. Natalia, Surfaceenhanced Raman spectroscopy of microorganisms: limitations and applicability on the single-cell level Analyst, 144 (2019) 943-953.

[29] M. Chisanga, H. Muhamadali, D.I. Ellis, R. Goodacre, Surface-Enhanced Raman Scattering (SERS) in Microbiology: Illumination and Enhancement of the Microbial World, Appl Spectrosc, 72 (2018) 9871000.

[30] P.C. Lee, D. Meisel, Adsorption and surface-enhanced Raman scattering of dyes on silver and gold sols, J.Phys.Chem, 86 (1982) 3391-3395.

[31] D. Tibor, Classification of Yeasts, CRC Press, Taylor \& Francis Group, New York, 2008.

[32] M. Moskovits, Surface selection rules J. Chem. Phys, 77 (1982) 4408.

[33] E. Thaillandier, J. Liquer, A. Taboury, Advances in Infrared and Raman Spectroscopy, in: C. RJH, H. RE (Eds.), Wiley, New York, 1985, pp. 65-81.

[34] P. W. Ranjith, G. Yoseph, Z. Lawrence D., On the difference between SERS spectra of cell growth media and whole bacterial cells, Appl Spectrosc, 65 (2011) 493-499. .

[35] P. W. Ranjith, L. Jean C., S.-B. Alexis, T. Roger, C. Catherine E., Z. Lawrence D., The biochemical origins of the surface enhanced Raman spectra of bacteria: metabolomics profiling by SERS, Anal Bioanal Chem, 408 (2016) 4631-4647.

[36] W.R. Premasiri, D.T. Moir, L.D. Ziegler, Vibrational fingerprinting of bacterial pathogens by surface enhanced Raman scattering (SERS), The International Society for Optical Engineering, 5795 (2005) 1929.

[37] Z. Sulin, G. Huajian, B. Gang, Physical Principles of Nanoparticle Cellular Endocytosis, ACS Nano, 9 (2017) 8655-8671. 
Graphical abstract

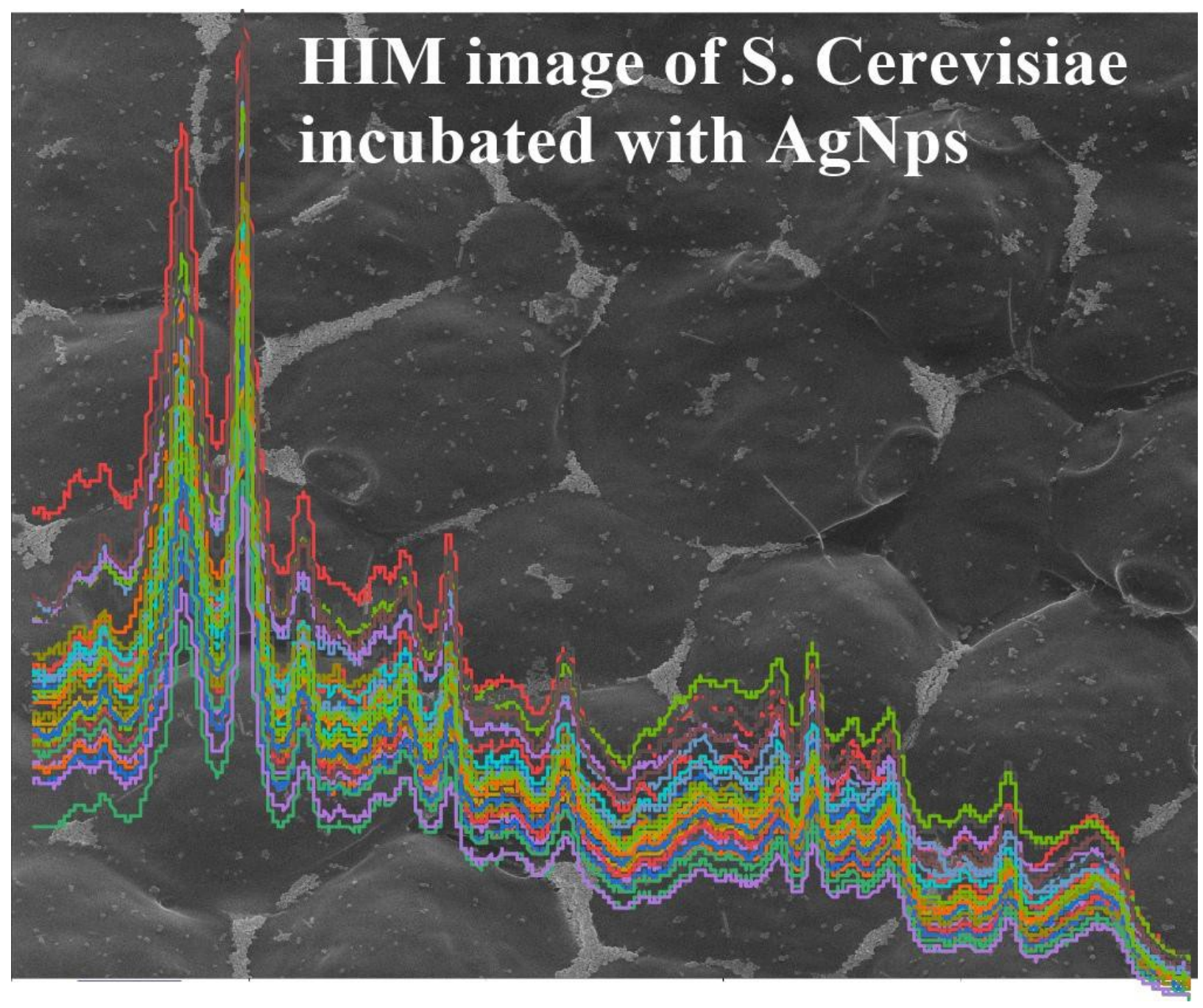




\section{Identifying yeasts using surface enhanced Raman spectroscopy}

\section{Tibebe Lemma ${ }^{1, *}$, Jin Wang ${ }^{2}$, Kai Arstila ${ }^{3}$, Vesa P. Hytönen ${ }^{4,}$ and J. Jussi Toppari ${ }^{3}$}

1 Faculdade de Clências e Tecnologia (FCT)-Universidade Estadual Paulista (UNESP)-Presidente Prudente, SP,19060-900, Brazil

2 Institute of Intelligent Machines, Chinese Academy of Sciences, Hefei, Anhui 230031, P. R. China

3 NanoScience Center, Department of Physics, P.O. Box 35 (YN), FI-40014, University of Jyväskylä, Finland

4 Faculty of Medicine and Health Technology and BioMediTech, Tampere University, Arvo Ylpön katu 34, FI-33520 Tampere, Finland and Fimlab Laboratories, Biokatu 4, FI-33520 Tampere, Finland

* Correspondence: tlemma@gmail.com (T.L.)

\section{Highlights:}

- High quality, i.e. sharp, reproducible, and excellent signal to noise ratio, SERS spectra of yeast microbes ((Saccharomyces cerevisiae (wild yeast), Dekkera bruxellensis and Wickerhamomyces anomalus obtained.

- SERS detection from very closely related fungal species (lysed and non-treated)

- Detection of the three species in complex beer medium

- High-resolution helium ion microscope (HIM) images give insight into the surface morphology of the yeast cells attached to AgNP

- SERS to be used for reliable detection and identification of the species in biosensing applications. 